\title{
IN VITRO INHIBITION OF BIOFILM FORMATION BY STAPHYLOCOCCUS AUREUS UNDER THE ACTION OF SELECTED PLANT EXTRACTS
}

\author{
Piegerová, A. ${ }^{1}$, Koščová, J. ${ }^{1}$, Schusterová, $P .{ }^{1}$, Nemcová, R. ${ }^{1}$, Kryvtsova, $M .{ }^{2}$ \\ ${ }^{1}$ Department of Microbiology and Immunology \\ University of Veterinary Medicine and Pharmacy, Komenského 73, 04181 Košice \\ Slovakia \\ ${ }^{2}$ Department of Genetics, Plant Physiology and Microbiology, Biological Faculty \\ Uzhhorod National University, Uzhhorod \\ Ukraine
}

jana.koscova@uvlf.sk

\begin{abstract}
In our study we investigated the ability of selected plant extracts to inhibit the formation of biofilms produced by Staphylococcus aureus. In the first phase, we focused on the optimisation of conditions for the correct method of an approach. For optimisation, we standardized the culture media and the bacterial culture in order to obtain interpretable results. The TSB (Tryptone Soya Broth) medium was used for the preparation of an inoculum from the bacterial suspension. For the in vitro tests of antibiofilm activity against the species Staphylococcus aureus CCM 3953, we used propylene glycol (PG) plant extracts from sage and rosemary, prepared in three different concentrations of $0.01 \%, 0.05 \%$ and $0.1 \%$. The tests were implemented in microtitre plates using crystal violet dye at $0.1 \%$ concentration for visualization of the intensity of a biofilm. The results were obtained, by spectrophotometric measurements at a wavelength of $550 \mathrm{~nm}$. Both rosemary and sage plant extracts had a significant effect on the formation of a biofilm by $S$. aureus. The antibiofilm activity was concentration-dependent as
\end{abstract}

the formation of biofilm was reduced more effectively with increasing concentration of the extracts. The best antibiofilm activity was observed with $0.1 \%$ rosemary extract resulting in $94 \%$ inhibition of the biofilm formation.

Key words: biofilm; inhibition; plant extracts; Staphylococcus aureus

\section{INTRODUCTION}

Microorganisms living in nature, in many cases survive only through a special form of an association, called biofilms. A biofilm is a community formed by different species of microorganisms reversibly attached to substrates, interphases, or to each other, while they are inserted in a matrix produced by them [5]. The main skeleton of biofilm is a structure consisting of exopolysaccharides (EPS), proteins in the form of enzymes, DNA or RNA, microbial cells and up to $95 \%$ of water. Some pathogenic microorganisms are capable of producing a biofilm as one of a number of 


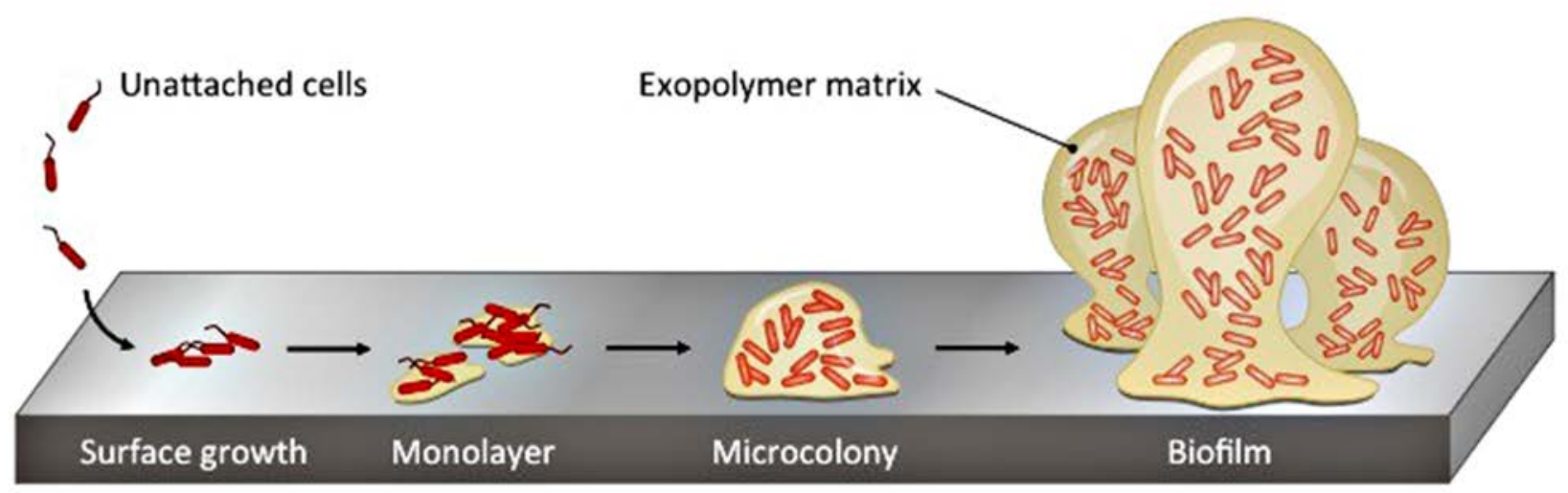

Fig. 1. Biofilm formation

Source: http://ib.bioninja.com.au/options/untitled/b3-environmental-protection/biofilms.html

defence mechanisms, resulting in their increased resistance to antimicrobials, particularly antibiotics [8]. Bacterial species important from the point of view of biofilm formation include those from the group "ESKAPE" (Enterococcus faecalis, Staphylococcus aureus, Klebsiella pneumoniae, Acinetobacter baumannii, Pseudomonas aeruginosa and Enterobacter spp.). These are bacteria that due to their multiresistance are frequently the agents of nosocomial infections. The infections with involvement of biofilm producing bacterial strains include for example periodontitis, valvular endocardititis, cystic fibrosis, urinary infections, dental plaques, infections of artificial joints and many others developing particularly in immunocompromised individuals [15]. Staphylococci belong to the bacteria that live in a natural habitat and are part of a microbiota of skin and mucous membranes of humans and other animals. On the other hand, pathogenic staphylococci may cause local or generalized infections and some of their strains have an ability to produce biofilms on both biotic and abiotic surfaces.

Biofilm formation is a very complex process during which the individually occurring cells form clusters and subsequently grow together. Formation of biofilms by nonmotile and motile bacterial species differs. If the conditions are favourable, expression of adhesins on the surface of non-motile bacterial species increases. This results in the increase of cohesion forces among cells and, subsequently in improved adhesiveness to the surface. On the contrary, motile species lose their motility and produce an extracellular matrix owing to which they produce aggregates and stay together. The matrix is a dynamic system in which a homeostasis is achieved and the organisation of this sys- tem allows the cells to use available nutrients. Four basic phases are recognised in the process of biofilm formation. In the first phase, which involves attachment to the surface, microbial cells adhere to the surface by means of fimbria or flagella. For the attachment they can also use physical forces such as van der Waals forces or electrostatic interactions [9].

After attachment and stabilization, the process of cell division starts. The initial signal that is controlled genetically, is the production of EPS. Microcolonies consist usually of a large number of the so-called micro-communities. They communicate among themselves in many ways. The coordinated bacterial growth is controlled by the so-called quorum sensing system (QS) utilizing signal proteins and other molecules for stimulation of growth of the microcolonies [11]. The QS allows bacteria to sense their own density and, subsequently, modify their phenotype [2].

The third phase involves the production of three-dimensional structure-microcolonies-and their maturation. This is related to the increased expression of biofilm formation associated genes. The products of these genes are inevitable for the production of exopolysaccharide, the main biofilm component. Another important part of the maturation of this microcolony is the production of extracellular matrix that is initiated immediately after attachment of bacteria to the surface.

The last phase is the potential detachment of colonies. There is a sequence of reactions which support the conversion of a bacterial colony to the motile form. Saccharolytic enzymes are produced to facilitate detachment of the colony aimed at re-colonization. The cells increase their expression of proteins responsible for the production of flagella 
so as to ensure motility of the detached bacteria. This detachment of the bacterial colony from the surface plays an important role in the spreading of the infections [9].

In staphylococci, the extracellular polysaccharide matrix with its principal component poly-N-acetyl-glucosamine is responsible for biofilm formation. The chemical structure of this polysaccharide corresponds to the structure of the polysaccharide intercellular adhesion (PIA). This was identified as the key component that enables the aggregation of staphylococci cells and their accumulation in the biofilm [17]. The synthesis of the PIA is encoded by the genes of the ica operone [13].

There exist substances, which are able to supress the production of a biofilm, even destroy the already formed biofilm. Metabolites of beneficial bacteria, disinfectants or plants extracts belong to the group of such substances.

Recently, some medicinal plants have increased the attention of researchers because of their potential and goaldirected ability to interfere with biofilms and thus facilitate the penetration of medicines to the bacterial agents. It was confirmed that, for example, lemon grass, oregano and Pimenta racemosa inhibit the formation of biofilms at concentrations $\leq 2 \%$. Also, rosewood, coriander, tea plant, Mentha $\times$ piperita and marjoram are effective at the same concentrations [7]. The components of Humulus lupulus L., such as lupulone, humulone and xanthohumol reduce the number of bacterial cells of Staphylococcus aureus released from biofilms. Extracts from Melaleuca alternifolia containing terpineole, terpinene-4-ol, or extracts from Melissa officinalis were more effective against biofilm produced by S. aureus and Escherichia coli than extract from Lavandula angustifolia containing lavender oil [3]. Currently implementation of nanoparticles acting as carriers of previously non-stabile essential oils help to interfere with bacterial biofilms. An example is a cinnamaldehyde obtained from cinnamon. When bound to nanoparticles, this aldehyde exhibits increased stability and thus also increased antibiofilm activity against methicillin-resistant Staphylococcus aureus (MRSA) strains and other bacteria [16].

The aim of this study was to investigate the antibiofilm activity of propylene glycol extracts of rosemary and sage, tested at various concentrations.

\section{MATERIALS AND METHODS}

The experiments were carried out using a reference, biofilm-producing strain Staphylococcus aureus CCM 3953. In order to obtain an inoculum, it was cultivated in meatpeptone broth (Himedia, India) for 18 hours at $37^{\circ} \mathrm{C}$ with constant mixing. This way the prepared night culture was subsequently diluted with a modified tryptone soya broth (TSB; Himedia, India) to $1 \%$ inoculum which was then used in an in vitro experiment.

We tested the antibiofilm activity of propylene glycol (PG) extracts prepared from the rosemary leaves (Rosmarinus officinalis L., PGR extract Certificate No. 310) and sage stems (Salvia officinalis L., PGS extract Certificate No. 309); both extracts were obtained from the company Calendula a. s., Nová Lubovňa, Slovakia). We prepared the base solutions of extracts in dimethyl sulphoxide (DMSO; SigmaAldrich, USA) at concentrations of $1 \%, 5 \%$ and $10 \%$ and diluted them 10-fold with TBS (Tris-buffered saline) to obtain concentrations $0.1 \%, 0.5 \%$ and $1 \%$.

The antibiofilm activity of the PG extracts were tested in standard 96-well microtitration plates (Greiner-BioOne, Austria) using a modified staining method according to O' T o o l e [14]. In the first step, we pipetted into individual wells $180 \mu \mathrm{l}$ of the bacterial suspension of staphylococci ( $1 \%$ inoculum). To the wells with staphylococci suspension, we added $20 \mu \mathrm{l}$ aliquots of the 10 -fold diluted solutions of extracts $(n=4)$ to obtain the following effective concentrations of extracts: $0.01 \%$; $0.05 \%$; and $0.1 \%$. We prepared also the respective negative controls by using $20 \mu \mathrm{l} 10 \%$ DMSO $(\mathrm{n}=4)$ instead of the diluted extracts. The plates were then incubated at $37^{\circ} \mathrm{C}$ for 24 hours. After the incubation, the supernatant was removed by aspiration and the individual wells with the formed bacterial biofilm were washed with distilled water 3-5 times, as needed. After thorough but careful washing, the biofilm was stained with $0.1 \%$ crystal violet solution $(200 \mu \mathrm{l}$ per well; $30 \mathrm{~min}$, room temperature). Then the crystal violet solution was aspirated again and wells were washed thoroughly with distilled water ( $3-5$ times). The following step consisted in a 10 -min extraction of the stain using $200 \mu \mathrm{l}$ of $30 \%$ acetic acid per well. After the action of the acidic solution, the content of each well was mixed with a pipette. The quantity of the stain obtained in this way was determined by measurement of the absorbance of each solution at $550 \mathrm{~nm}$ employing a spectrophotometer Synergy HT (Biotek, USA). 


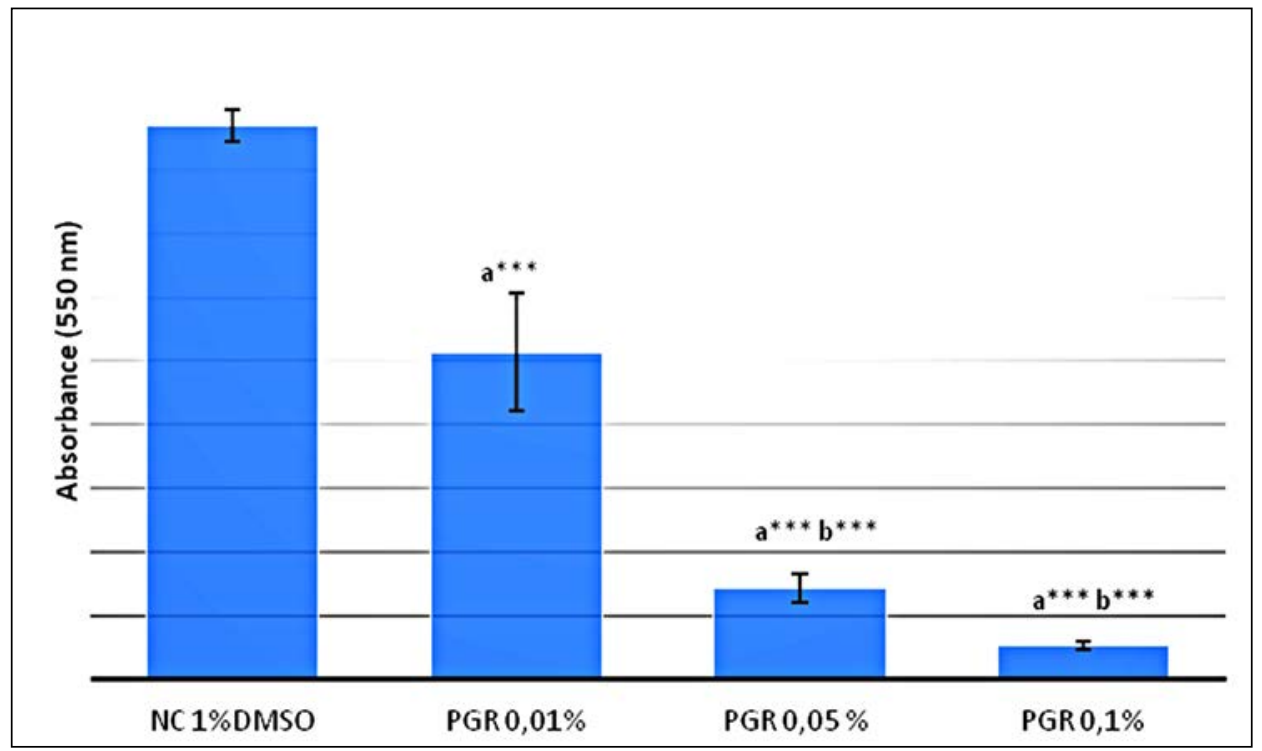

Fig. 2. Inhibition of $S$. aureus 3953

Biofilm by propylene glycol extract of rosemary $(n=4)$. PGR — propylene glycol rosemary extract; NC—negative control in DMSO; $\mathrm{a}$-significantly different from NC; b-significantly different from $0.01 \%$; $c$-significantly different from $0.05 \%$; ${ }^{* *}-\mathrm{P}<0.001$

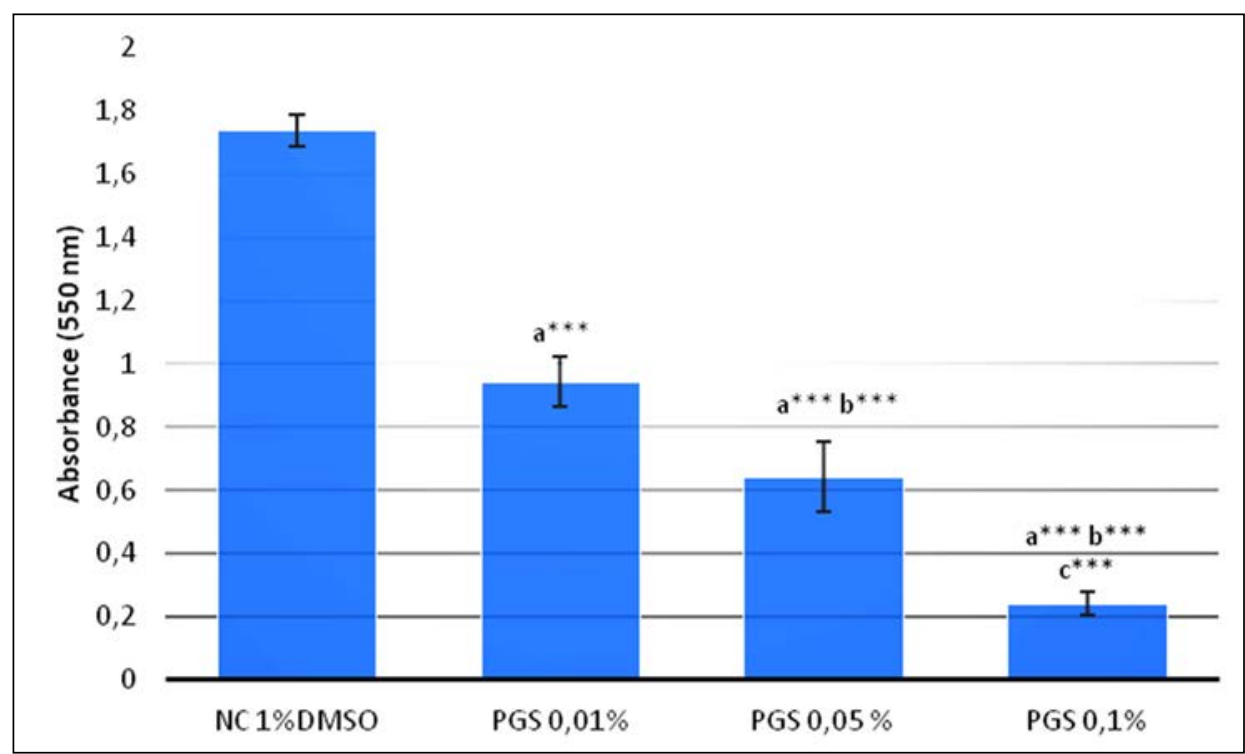

Fig. 3. Inhibition of S. aureus 3953

Biofilm by propylene glycol extract of sage $(n=4)$. PGS- propylene glycol sage extract; NC-negative control in DMSO; a - significantly different from NC; b-significantly different from $0.01 \%$; $c$-significantly different from $0.05 \%$; ${ }^{* *}-\mathrm{P}<0.001$

For statistical analysis of the results, a 1-way ANOVA was used. Significant differences between groups were assessed by Tukey's test. The difference between various concentrations of the plant extracts were determined by the unpaired t-test. P-values $<0.05$ were considered significant.

\section{RESULTS AND DISCUSSION}

The results of our experiments indicated that the antibiofilm activity of the PG extracts of rosemary and sage was concentration dependent as shown in Figs. 2 and 3. With 
increasing concentration of plant extracts the biofilm formation by Staphylococcus aureus decreased. The PG extracts of both plants showed the highest anti-biofilm concentration at a concentration of $0.1 \%$, followed by concentrations $0.05 \%$ and $0.01 \%$; however, the antibiofilm action of the sage extract was lower compared to the rosemary extract $(\mathrm{P}<0.0001)$. While the inhibition of biofilm formation by the sage extract ranged from $46 \%$ (0.01\% concentration) to $86 \%(0.1 \%$ concentration), the rosemary extract caused $96 \%$ inhibition at $0.1 \%$ concentration and $46 \%$ at $0.01 \%$ concentration.

An antibiofilm activity of PG extract of rosemary was reported also by O live i $\mathrm{r}$ a et al. [6]. These authors observed that the extract significantly reduced the growth and multiplication of bacteria (CFU per $\mathrm{ml}$ ) in monomicrobial biofilms of Staphylococcus aureus. The extracts of concentration $2 \%$ significantly reduced biofilm within 5 minutes. Better results in comparison with biofilms produced by S. aureus were obtained with the strains Candida albicans and Pseudomonas aeruginosa. The above study used extracts prepared by similar procedure as used in our study. However, the highest inhibition of biofilm formation by PG extract of rosemary was reached with extract with a much lower concentration (0.1\%).

The extract of rosemary at a concentration of $25 \mu \mathrm{l} . \mathrm{ml}^{-1}$ reduced the biofilm produced by Staphylococcus epidermidis by $57.1 \%$. At lower concentration $\left(12.5 \mu \mathrm{l} . \mathrm{ml}^{-1}\right)$, rosemary decreased adhesion of cells by $49.21 \%$ but reduced the formation of biofilm by only $29.05 \%$ [10]. The rosemary extract tested in this study had an antibiofilm affect similar to that in our experiments but the inhibition ranged from $46 \%$ to $86 \%$.

In the study by $\mathrm{Ce} y \mathrm{la} \mathrm{n}$ [4], the antibiofilm activity of the rosemary extract against the biofilm produced by various bacteria including $S$. aureus was tested and the authors reported that the rosemary extract was the most effective specifically against $S$. aureus produced biofilm. The author used a longer cultivation of the inoculum ( $48 \mathrm{~h}$ ) in comparison with our method. The inhibition observed was $60.76 \%$.

In addition to propylene glycol extract, other types of plant extracts also inhibited the formation of biofilms. Hydroalcohol extract of rosemary showed anti-adherent action and decreased the formation of biofilms [12].

A l - B a k r i et al. [1] investigated the antibiofilm effect of ethanol extracts from sage and sage essential oil. Into individual wells of microtitration plates they added $100 \mu \mathrm{l}$ of extract and $10 \mu \mathrm{l}$ of staphylococci inoculum, so the final volume of the well content was smaller than that used in our study which explains the higher anti-biofilm activity reached by these authors. The extract from sage resulted in $86.2 \%$ and $83.4 \%$ inhibition of the production of biofilm by MRSA (methicillin-resistant S. aureus) strain and a $98.3 \%$ inhibition of $S$. aureus.

The results of several studies are in agreement with our observations that indicated higher anti-biofilm activity of rosemary extract in comparison with sage extract. Also the percentage of inhibition achieved by rosemary extracts was similar or were in the range observed in our study taking into consideration the concentration of the tested extracts. However, standardization of the procedures appears important for reproducibility of results as different conditions of incubation and preparation of relevant inocula applied to microtitration plates influence the investigated activity.

\section{CONCLUSIONS}

From the medical point of view, the formation of biofilms by pathogenic microorganisms on both biotic and abiotic surfaces is an undesirable phenomenon with serious implications. The standardization of procedures is important in order to reach creditable interpretable results. Some compounds of natural origin, for example those in certain plant extracts, have the capacity to inhibit the formation of such biofilms. Our study confirmed the antibiofilm concentration-dependent effectiveness of rosemary and sage propylene glycol extracts with the highest activity of rosemary extract at concentration of $0.1 \%$.

\section{ACKNOWLEDGEMENT}

The study was supported by the project APVV-15-0377.

\section{REFERENCES}

1. Al-Bakri, A. G., Othman, G., Afifi, F. U., 2010: Determination of the antibiofilm, antiadhesive, and anti-MRSA activities of seven Salvia species. Pharmacognosy Magasine, 6 (24), 264-270. DOI: 10.4103/0973-1296.71786. 
2. Arciola, C. R., Campoccia, D., Ravaioli, S., Montanaro, L., 2015: Polysaccharide intercellular adhesin in biofilm: structural and regulatory aspects. Front. Cell Infect. Microbiol., 5, 7, PMID: 25713785. DOI: 10.3389/fcimb.2015.00007.

3. Budzyńska, A., Wieckowska-Szakiel, M., Sadowska, B., Kalemba, D., Rozalska, B., 2011: Antibiofilm activity of selected plant essential oils and their major components. Polish Journal of Microbiology, 60 (1), 3541.

4. Ceylan, O., 2014: The in vitro antibiofilm activity of Rosmarinus officinalis L. essential oil against multiple antibiotic resistant Pseudomonas sp. and Staphylococcus sp. Journal of Food, Agriculture and Environment, 12 (3-4), 82-86.

5. Charaklis, W. G., Marshall, K. C. (Eds.), 1990: Biofilms. John Wiley and Sons, New York, 796 pp.

6. de Oliveira, J. R., de Jesus, D., Figueira, F. E., de Oliveira, F. E., Pacheco Soares, C. P. Camargo, S. E. A., et al., 2017: Biological activities of Rosmarinus officinalis L. (rosemary) extract as analysed in microorganisms and cells. Exp. Biol. Med., 242 (6), 625-634. DOI: 10.1177/1535370216688571.

7. Hammer, K. A., Carson, C. F., Riley, T. V., 1999: Antimicrobial activity of essential oils and other plant extracts. J. Appl. Microbiol., 86 (6), 985-990.

8. Høiby, N., Bjarnsholt, T., Givskov, M., Molin, S., Ciofu, O., 2010: Antibiotic resistance of bacterial biofilms. Int. J. Antimicrob. Agents, 35 (4), 322-332. https//hal.archives-ouvertes.fr/ hal-00567285.

9. Jamal, M., Ahmad, W., Andleeb, S., Jalil, F., Imran, M., Nawaz, M. A., et al., 2017: Bacterial biofilm and associated infections. Journal of the Chinese Medical Association, 81 (1), 7-11. DOI: 10.1016/j.jcma.2017.07.012.

10. Jardak, M., Elloumi-Mseddi, J., Aifa, S., 2017: Chemical composition, anti-biofilm activity and potential cytotoxic effect on cancer cells of Rosmarinus officinalis L. essential oil from Tunisia. Lipids Health Dis., 16, 190. DOI: 10.1186/ s12944-017-0580-9.
11. Kumar, A., Alam, A., Rani, M., Ehtesham, N. Z., Hasnain, S. E., 2017: Biofilms: Survival and defense strategy for pathogens. Int. J. Med. Microb., 307 (8), 481-489. DOI: 10.1016/j. ijmm.2017.09.016.

12. Nogueira, J., Costa, R., da Cunha, M., Cavalcante, T., 2017: Antibiofilm activity of natural substances derived from plants. African Journal of Microbiology Research, 11 (26), 1051-1060. DOI: $10.5897 / A J M R 2016.8180$.

13. O'Gara, J. P., 2007: ica and beyond: biofilm mechanism and regulation of Staphylococcus epidermidis and Staphylococcus aureus. FEMS Microbiol. Lett., 270 (2), 179-88. DOI:10.1111/ j.1574-6968.2007.00688.x.

14. O’Toole G. A., 2011: Microtiter dish biofilm formation assay. J. Vis. Exp., 47. http://www.jove.com/details.php?id=2437. DOI: $10.3791 / 2437$.

15. Rabin, N., Zheng, Y., Opoku-Temeng, C., Du, Y., Bonsu, E., Sintim, H. O., 2015: Biofilm formation mechanisms and targets for developing antibiofilm agents. Future Medicinal Chemistry, 7 (4), 493-512. DOI: 10.4155/fmc.15.6.

16. Ramasamy, M., Lee, J. H., Lee, J., 2017: Direct one-pot synthesis of cinnamaldehyde immobilized on gold nanoparticles and their antibiofilm properties. Colloids and Surfaces B Biointerfaces, 160, 639-648. DOI: 10.1016/j.colsurfb.2017.10.018.

17. Rohde, H., Frankenberger, S., Zahringer, U., Mack, D., 2010: Structure, function and contribution of polysaccharide intercellular adhesin (PIA) to Staphylococcus epidermidis biofilm formation and pathogenesis of biomaterial-associated infections. Eur. J. Cell Biol., 89 (1), 103-11. DOI: 10.1016/j. ejcb.2009.10.005.

Received November 10, 2018

Accepted March 8, 2019 